\title{
Numerical Simulations of Reactive Two-Phase Flows in Interior Ballistics
}

\author{
Roman Wölbing, Maxime Chiroli, Claude Steinbach, Robert Hruschka, Barbara Baschung \\ French-German Research Institute of Saint-Louis, ISL \\ 5 rue du Général Cassagnou, 68300 Saint-Louis, France \\ roman.woelbing@isl.eu; maxime.chiroli@isl.eu; claude.steinbach@isl.eu; robert.hruschka@isl.eu; \\ barbara.baschung@isl.eu
}

\begin{abstract}
The present paper is devoted to three-dimensional numerical simulations of reactive two-phase flows in interior and transitional ballistics. The model presented takes a variety of phenomena into account such as mass transfer by the combustion of complex grain geometries, drag force experienced by the propellant bed in the gas flow, and convective and radiative heat transfer between the solid and the gaseous phase to simulate the process of ignition. A multi-dimensional finite volume scheme based on a nonconservative two-velocity one-pressure model is deployed to compute the pressure distribution in a $40 \mathrm{~mm}$ weapon system, the velocity of the projectile, and the flow field of the muzzle flash. Comparisons between the obtained results and experimental findings, as well as the predictions of state-of-the-art lumped-parameter codes, show good agreement and serve as a validation. Finally, interior ballistics simulations involving novel grain geometries are discussed which exhibit more effective combustion properties as compared to conventional grains and thus lead to enhanced performances.
\end{abstract}

Keywords: Interior Ballistics, Combustion, Numerical Simulation, Two-Phase Flow, Propellant Grain Geometry.

\section{Introduction}

Propellant-based weapon systems have been used since at least the $13^{\text {th }}$ century and still form the base of any safety authority or military effort [1]. Research, however, is often expensive and dangerous for all parties involved, not only in the case of development or optimization of new devices [2] but also in terms of designing countermeasures [3], such as bullet proof vests or armoured vehicles. For this reason, a lot of work has been invested over the past decades into the creation of numerical models and codes to simulate the complex physical and chemical phenomena occurring in interior ballistics (IB). This resulted in a wide range of software applications, such as zero-dimensional (0D) lumped-parameter codes like STANAG [4] or IBHVG2 [5] on the one hand, and multi-dimensional computational fluid dynamics (CFD) codes like FHIBS [6], MOBIDIC [7] or Casbar [8] on the other hand. Likewise, it was shown that IB can be successfully implemented into commercially available software packages like ANSYS FLUENT [9] as well as open source environments like OpenFOAM [10]. The common goal of all these efforts is to accurately predict the combustion of the propellant, the pressure evolution in the gun and the velocity of the projectile at the muzzle.

The underlying process of IB can be subdivided into three, frequently overlapping, stages. 1) The primer, usually black powder, is initiated in an ignition module, generating hot gases which enter the combustion chamber and heat up the propellant bed until the ignition temperature is reached. 2) The propellant grains start to burn leading to a steep rise in pressure and temperature within the gun. While the burning of most energetic materials is in first order approximation independent of temperature, the rise in pressure results in a roughly linear increase in the combustion rate. 3) At a sufficiently high pressure the projectile begins to move and the available volume for further combustion increases which is usually accompanied by a motion of the propellant residuals towards the projectile base and thus by a drop in pressure at the breech of the gun. The IB cycle ends as soon as the projectile reaches the muzzle.

Here, we show three-dimensional (3D) simulations of the IB of a 40mm system with the ISL code Axisymmetric Model of Interior Ballistics (AMI) [11], [12] involving all of the aforementioned phases. The theoretical model is briefly presented in section 2 and the results of the calculations are discussed and compared to the corresponding measurements in section 3. Furthermore, we demonstrate that the scope of the model can be extended to additionally compute the fluid 
dynamics of transitional ballistics effects, such as the muzzle flash. In section 4, the 3D combustion module of the code is described in detail and novel grain geometries are discussed.

\section{Theoretical Model}

Since the theoretical background has already been extensively elaborated in previous works [13]-[15], this section only addresses the most crucial points of the model. AMI is based on a finite volume scheme which employs rectilinear grids for one-dimensional (1D) and two-dimensional (2D) computational domains and curvilinear grids in the $3 \mathrm{D}$ case. Without loss of generality, only the latter case is discussed, see Fig 1.

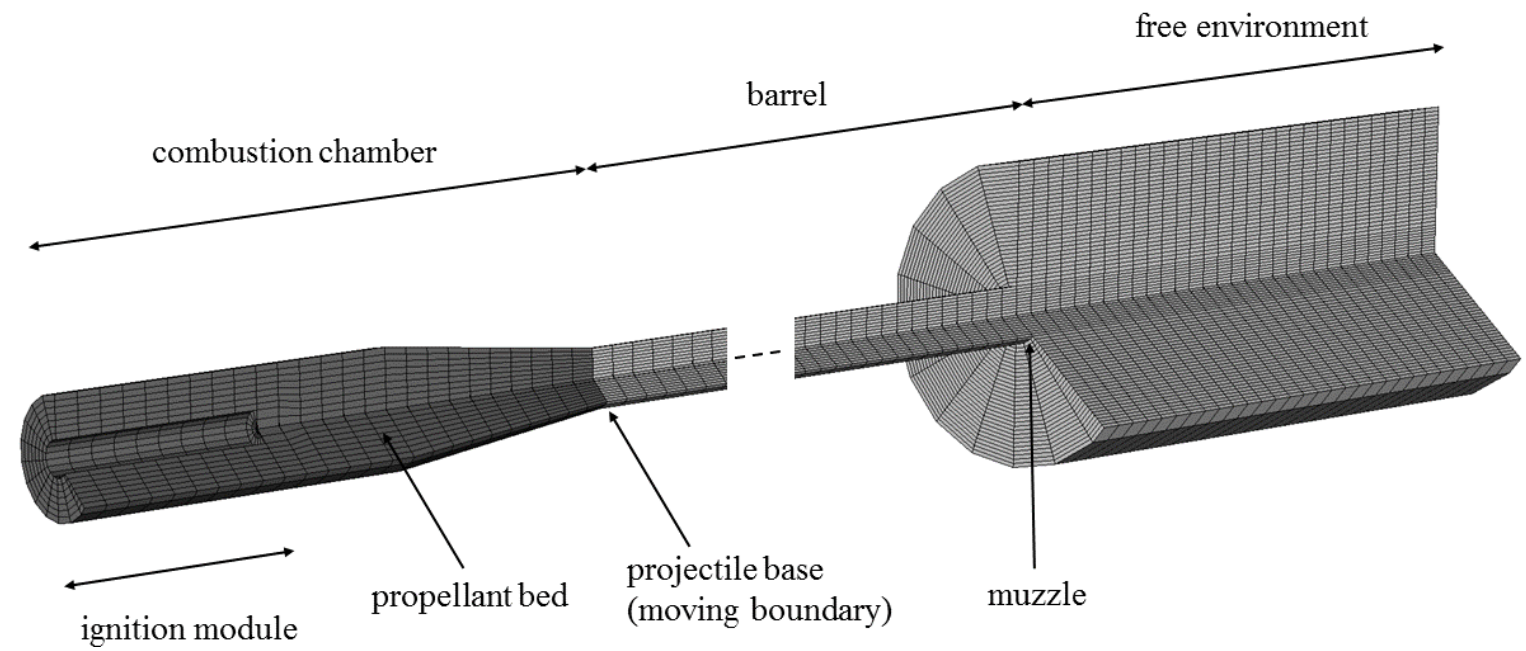

Fig. 1: Example of an edited mesh deployed for 3D simulations. The computational domain consists of an ignition module embedded in the combustion chamber containing the propellant (dark grey). The projectile is modelled by a 2D moving boundary separating the chamber and the barrel (light grey). An additional mesh part is added next to the muzzle representing atmospheric ambient conditions.

The ignition module at the bottom of the combustion chamber contains the primer from which hot gas enters the propellant bed via predefined mesh faces resembling perforation holes. In the chamber, both the gaseous and the solid propellant phase are treated as interpenetrating continuous media. The projectile, separating chamber and barrel, is described by a 2D wall-like boundary which runs through the domain according to the pressure in the weapon. After the muzzle, a further mesh part is added for simulations of the muzzle flash at the time the projectile leaves the barrel.

In order to model the reactive flow, the two phases are considered to be governed by the Euler equations, i.e. by conservation of mass, momentum and energy, complemented by two convection equations representing the time evolution of the enthalpy and the burned thickness of the propellant grains. A source term is added to the system of equations for taking into account the specific characteristics of IB, such as mass transfer rate from the solid to the gaseous phase via Vieille's law, chemical energy deposited in the system by the combustion of the energetic materials, drag force experienced by the grains in the gas flow [16], as well as convective and radiative heat transfer between both phases to model the process of ignition [17]. As mass transfer rate, drag force and ignition delay are highly sensitive to the specific grain surface, the latter is computed by a 3D module which also allows for the inclusion of slivering effects and more complex grain geometries, e.g. co-layered propellants [18]. The combustion products are assumed to be equivalent to the initial gas phase and to follow the Nobel-Abel equation of state. Finally, the governing equations are solved by an approximate Godunov scheme adapted to systems of differential equations involving nonconservative terms [19].

\section{40mm Gun}

We compare 3D simulations on a 40mm gun system to experimental measurements and to results of the lumpedparameter code STANAG 4367. Tab. 1 summarizes only the most relevant data used as input for the calculations. 
Table 1: Most relevant input data used for the simulations with AMI and STANAG 4367.

\begin{tabular}{|l|l|}
\hline Combustion chamber length & $0.37 \mathrm{~m}$ \\
\hline Combustion chamber diameter & $58 \mathrm{~mm}$ \\
\hline Barrel length & $4.11 \mathrm{~m}$ \\
\hline Barrel caliber & $40 \mathrm{~mm}$ \\
\hline Projectile mass & $279 \mathrm{~g}$ \\
\hline Propellant mass & $400 \mathrm{~g}$ \\
\hline Propellant density & $1.59 \mathrm{~g} / \mathrm{cm}^{3}$ \\
\hline Explosive heat of propellant & $4300 \mathrm{~J} / \mathrm{g}$ \\
\hline Propellant co-volume & $1030 \mathrm{~cm}^{3} / \mathrm{g}$ \\
\hline Propellant geometry & $7 \mathrm{hole} \mathrm{cylindrical}^{3}$ \\
\hline Primer mass & $3 \mathrm{~g}$ \\
\hline Primer density & $1.75 \mathrm{~g} / \mathrm{cm}^{3}$ \\
\hline Explosive heat of primer & $2840 \mathrm{~J} / \mathrm{g}$ \\
\hline Primer co-volume & $1084 \mathrm{~cm}^{3} / \mathrm{g}$ \\
\hline Resistive pressure of projectile & $12 \mathrm{MPa}$ \\
\hline
\end{tabular}

Fig. 2a) shows the pressure evolution within the weapon as obtained by the numerical calculations with AMI and STANAG 4367 along with the experimental findings. In Fig. 2b) we compare the velocity of the projectile as a function of time. Note that only the muzzle velocity is available from the test firings.
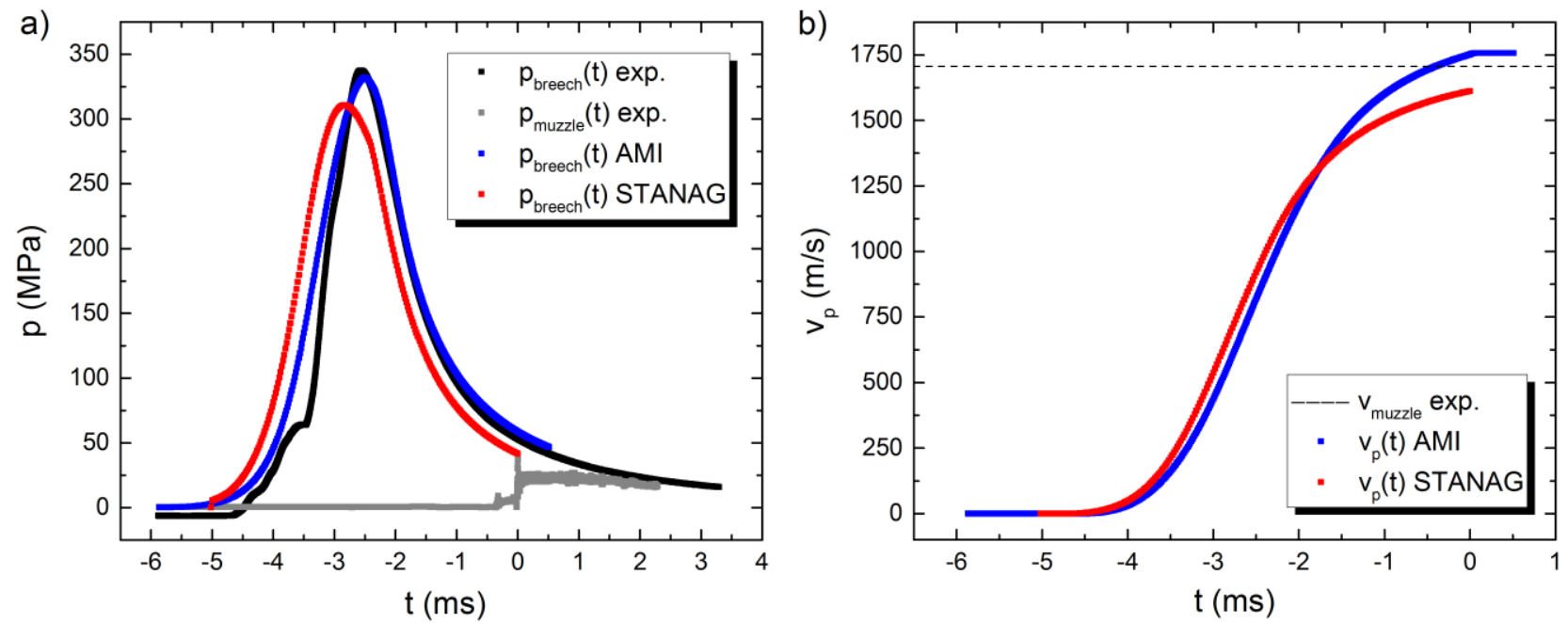

Fig. 2: a) Evolution of pressure within the 40mm gun measured at the breech (black dots) and at the muzzle (grey dots). Blue and red dotted curves give the results of the simulations with AMI and STANAG, respectively. b) Projectile velocity as a function of time as calculated with AMI (blue dots) and STANAG (red dots). Black dashed line is the projectile velocity at the muzzle obtained from the experiment.

In order to compare the results, the time scale for all curves has been adjusted so that the zero point coincides with the moment the projectile reaches the muzzle. In the experiment this moment is indicated by a peak in pressure close to the muzzle, see grey dots in Fig 2a). We find very good agreement between the pressure measurement at the breech (black dots) and the simulation with AMI (blue dots), while STANAG underestimates maximum pressure as well as shot time (red dots). For the projectile velocity, we find qualitative agreement between AMI and STANAG, see Fig. 2b) blue and red dots, respectively. As compared to the measured velocity at the muzzle of $1707 \mathrm{~m} / \mathrm{s}$ (black dashed line), we find deviations of $2.7 \%$ for AMI and $5.9 \%$ for STANAG. The reason for this is currently not understood but might be connected to a 
failure of the engraving mechanism when the projectile begins to move. We define the ignition delay as the period from the initialization of the primer to the time the pressure in the chamber has reached 5MPa. With this we find an ignition delay of $12.29 \mathrm{~ms}$ from the experimental data and $10.05 \mathrm{~ms}$ for the simulation of AMI (not shown in Fig. 2a)). The large deviation might be caused by several factors. On the one hand, many quantities important to the ignition of energetic materials are unknown, e.g. the radiative emission factor of the grains. On the other hand, primers, especially black powder, often exhibit deflagration which is currently not possible to model within AMI. The process of ignition is not taken in account in STANAG.

As previously stated, phenomena belonging to the field of transitional ballistics can also be simulated in AMI by extending the computational domain behind the muzzle as in for example [20]. For the 40mm system, the additional part of the mesh is 7 calibers in diameter and $0.9 \mathrm{~m}$ in length. We assume zero-gradient boundary conditions for the patch faces of this domain. Fig. 3 shows a pseudo-colour pressure distribution in the free environment behind the muzzle for four points in time after the projectile left the barrel.

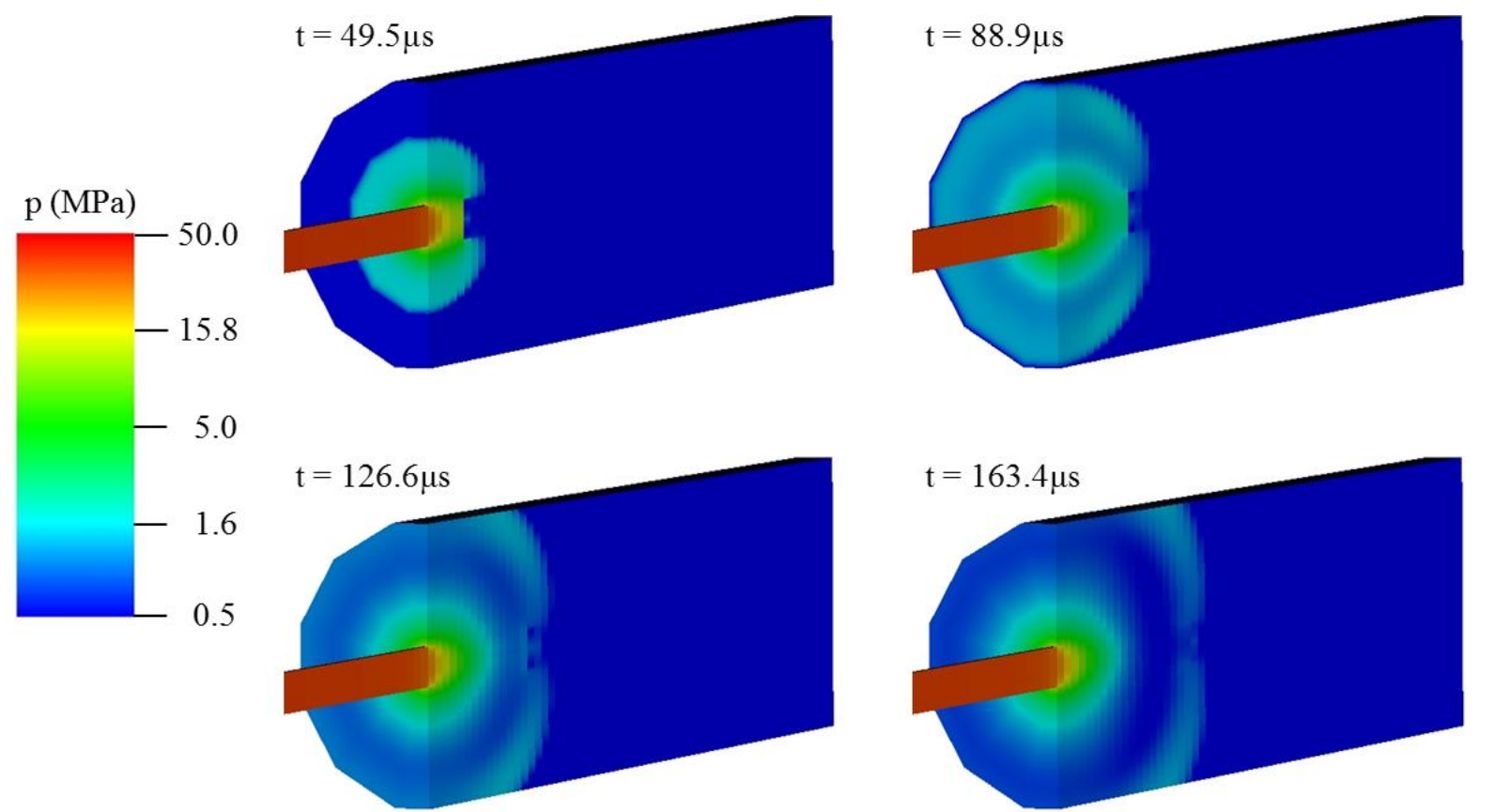

Fig. 3: Pseudo-colour pressure distribution (log scale) of the muzzle flash for four consecutive points in time after the projectile left the barrel [21]. To provide a better view, only half of the computational domain is shown.

As soon as the projectile leaves the muzzle, the highly compressed propellant gas exits the gun in a supersonic jet to the sides of the bullet (Fig.3; top left). In this stage, the projectile experiences a small increase in acceleration due to the expanding gas until it is slowed down by aerodynamic drag in the further stages. Subsequently, a high-temperature blast wave $(T \approx 1500 \mathrm{~K})$ is developed from the muzzle flash (top right). In the latter stages, blast wave and projectile separate from the muzzle flash at roughly equal velocity (bottom left and right).

So far, the results of the transitional ballistics simulations can only be compared with measurements of the pressure close to the muzzle of the $40 \mathrm{~mm}$ gun, i.e. the grey dotted curve in Fig. 2a), for which we find reasonable agreement. Further analysis will be the subject of future work. It should be noted, however, that the current state of the code does not involve the Navier-Stokes equations and therefore effects of turbulence are neglected. Also, in order to have a better picture of the muzzle flow and its interaction with the bullet, precursor flow and more complex projectile geometries should be considered in the future. 


\section{Optimized Grain Geometries}

Most IB codes only consider standard propellant geometries, e.g. spherical or multi-hole cylindrical grains. With the advent of 3D printing techniques however, high yield production of more complex geometries seems feasible [18], [22]. To study the impact of the shaping of grains on pressure evolution and projectile velocity, we deploy a 3D combustion algorithm in AMI. First, the initial geometry of the object is constructed and thereafter meshed by using a Delaunay triangulation scheme. From this surface $S$ and volume $V$ are calculated. Secondly, the object is downsized by reducing its characteristic length scale by a predefined fraction assuming a burning perpendicular to the grains surface. The process is repeated as long as surface and volume remain positive numbers. Fig. 4a) depicts three instants of the burning of the sevenhole cylindrical grain (left column) used for the calculations in the previous section.

a)

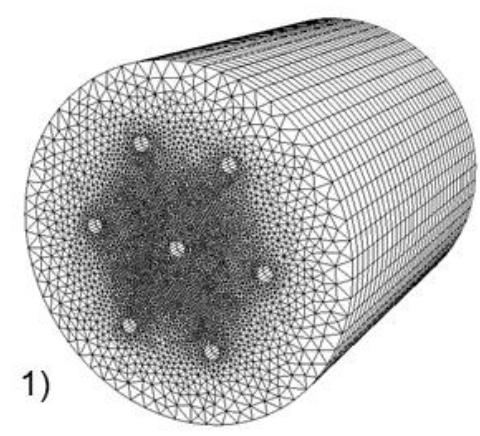

2)

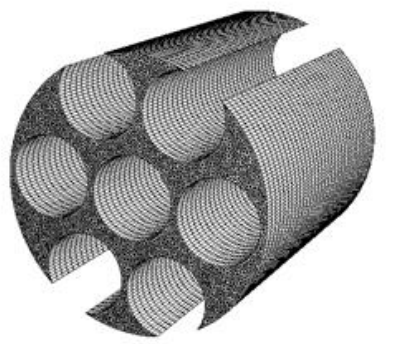

3)

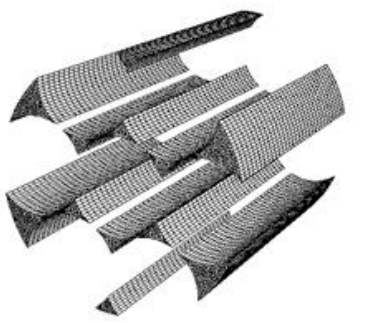

i)

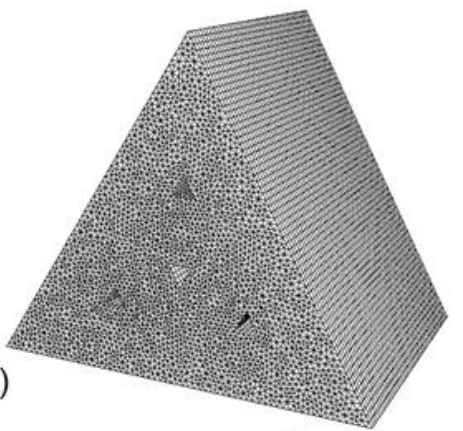

ii)

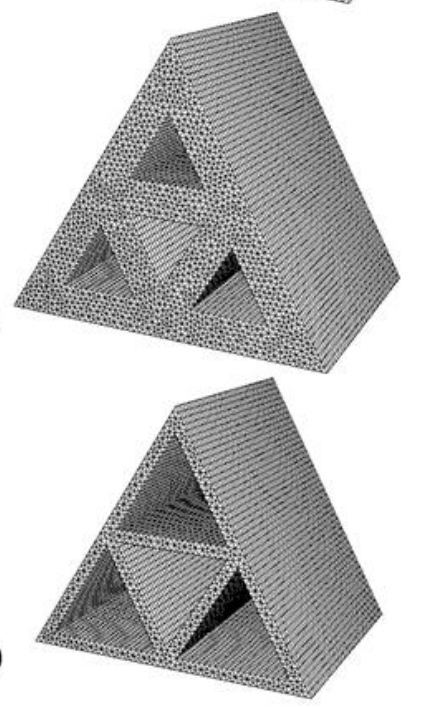

b)

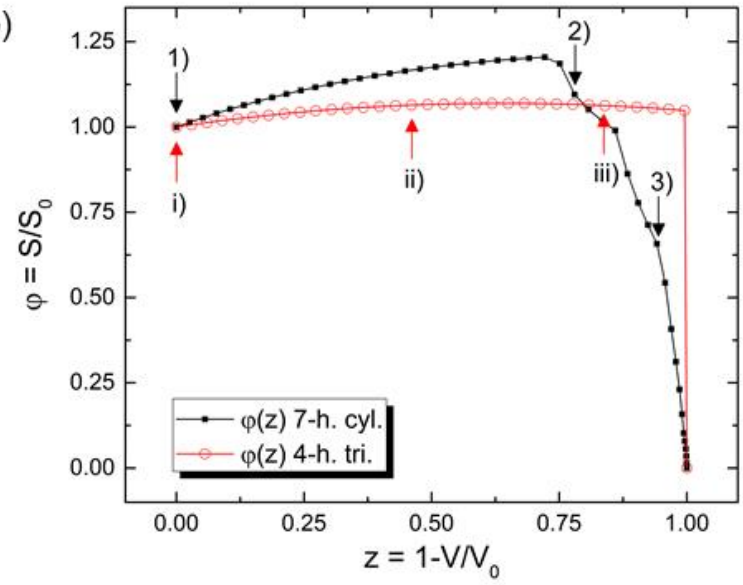

c)

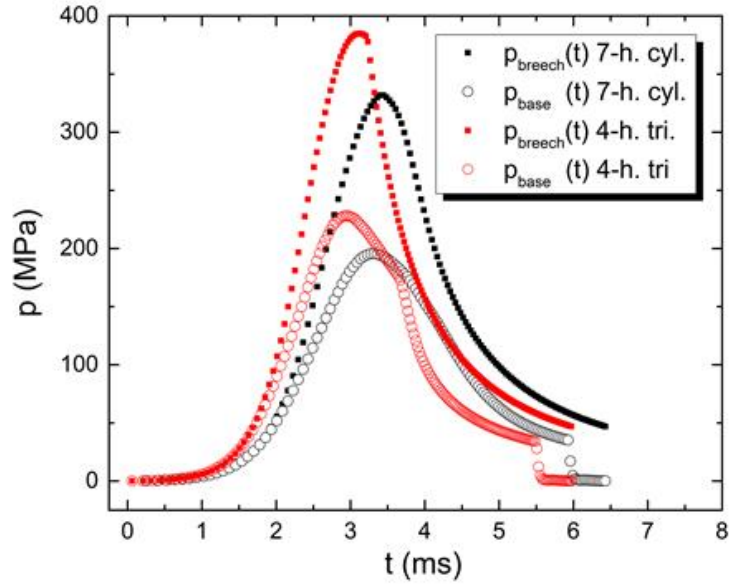

Fig. 4: a) Three instants of the combustion of a seven hole cylindrical grain (left column) and of a four hole extruded triangle (right column). Numbering corresponds to b). b) Form function for the seven-hole cylindrical grain (black dotted line) and for the four hole extruded triangle (red dotted line). c) IB cycle simulations for the $40 \mathrm{~mm}$ system calculated with AMI for both grain types. Shown are pressure at the breech and the projectile base for a propellant bed comprising seven hole cylindrical grains (black dots) and four hole extruded triangles (red dots).

In Fig. 4b) we show the obtained form function $\varphi(z)$ for this grain type (black dotted line), which is the instantaneous surface $S$ normalized to the initial surface $S_{0}$ as a function of the burned fraction $z$. Numbers correspond to the instants shown in a). The grain exhibits progressive burning, i.e. its surface increases during the process, until the sliver point is reached at a mass fraction of roughly $75 \%$. From now on the breaking up of the object results in small residuals with poor surface-to-volume ratios which is unfavourable for the purpose of IB. In the right column of Fig. 4a) three instants of the burning of a four-hole extruded triangle are shown. This geometry is inspired by the second iteration of a Sierpinski 
triangle and is constructed to match the volume of the seven-hole cylindrical grain. The form function of the Sierpinski-like grain is less progressive, however without transition to slivering, see red dotted line in Fig. 4b).

To investigate the impact of the optimized shape, we carry out the simulation of the 40mm gun with the same parameters as in section 3. Note that since the volumes of both grain types are identical, not only the propellant charges but also the number of grains are equal for both calculations. In Fig. 4c) the pressure evolution at the breech and at the base of the projectile are shown for propellant beds comprising seven hole cylindrical grains (black dotted) and four hole triangular grains (red dotted). In the latter case, the onset of the increase in pressure occurs earlier since the initial surface of the Sierpinski-like grains is approximately $16 \%$ larger as compared to the cylindrical shaped grains. This leads to higher maximum pressure and a shorter overall shot time. For the muzzle velocity of the projectile, we find $1793 \mathrm{~m} / \mathrm{s}$, which is an increase of $2.3 \%$ with respect to the previous results. Finally, we note that although the ignition of the optimized geometry has not yet been studied, the time delay should be significantly shorter due to the higher surface.

\section{Conclusion}

In conclusion, simulations of the complete IB cycle of a $40 \mathrm{~mm}$ weapon system were conducted using the multidimensional reactive two-phase flow software AMI. In general, the results were found to be in good agreement with measurements of pressure and overall shot time with only minor variances of around $1 \%$. For the onset of increase in pressure, slightly larger deviations are observed, which indicate that the static/dynamic friction between projectile and barrel is insufficiently modelled and needs to be reviewed. It might be reasonably assumed that the deviation in muzzle velocity of currently $2.7 \%$ can be further reduced by this measure. By computing the pressure distribution close to the muzzle of the $40 \mathrm{~mm}$ gun after the projectile leaves the barrel, it was further shown that transitional ballistics effects can also be, at least qualitatively, described by the model. It was demonstrated that the $3 \mathrm{D}$ combustion scheme within AMI is able to predict the burning of standard and more complex shaped grains. A novel shape, inspired by a fractal geometry, was proposed which avoids the negative impact of slivering entirely.

The model described in this study can still be considerably improved. Future work will focus on the chemical kinetics of the combustion process of the propellant, as well as on different ignition mechanisms, e.g. ignition by the injection of a plasma jet into the combustion chamber.

\section{References}

[1] A. W. Horst, "A Brief Journey Through the History of Gun Propulsion," ARL-TR-3671, U.S. Army Research Laboratory, Aberdeen Proving Ground, US-MD, 2005.

[2] D. Åberg, P. Hermansson, A. Sättler and D. Rakus, "Plasma Ignition Response for LOVA Gun Propellant at Low Loading Densities," IEEE Transactions on Plasma Science, vol. 43, no. 5, pp. 1316-1320, 2015.

[3] M. Seidl, T. Wolf and R. Nuesing, "Numerical Investigations on Ricochet of a Spin-Stabilised Projectile on Differently Shaped Target Surfaces," in Proceedings of the $30^{\text {th }}$ International Symposium on Ballistics, Long Beach, US-CA, 2017, pp. 2299-2311.

[4] "Thermodynamic Interior Ballistic Model with Global Parameters," NATO North Atlantic Council, PFP(NAAG)D(2009)0011, STANAG 4367, 2009.

[5] R. D. Anderson and K. D. Fickie, "IBHVG2 - A User's Guide," ARL-TR-2829, U.S. Army Research Laboratory, Aberdeen Proving Ground, US-MD, 1987.

[6] C. R. Woodley, S. Billet, C. Lowe, W. Speares and E. Toro, "The FHIBS internal ballistics code," in Proceedings of the $22^{\text {nd }}$ International Symposium on Ballistics, Vancouver, Canada, pp. 295-302, 2005.

[7] B. Longuet, P. D. Pieta, P. Franco, G. Legeret, A. Papy, D. Boisson, C. Reynaud, P. Millet, E. Taiana and A. Carrere, "MOBIDIC-NG: A 1D/2D CFD Code Suitable for Interior Ballistics and Vulnerability Modelling," in Proceedings of the $22^{\text {nd }}$ International Symposium on Ballistics, Vancouver, Canada, pp. 362-371, 2005.

[8] R. J. Gollan, I. A. Johnston, B. T. O'Flaherty and P. A. Jacobs, "Development of Casbar: a Two-Phase Flow Code for the Interior Ballistics Problem," in Proceedings of $16^{\text {th }}$ Australasian Fluid Mechanics Conference, Gold Coast, Australia, 2007, pp. 295-302.

[9] A. Bougamra and H. Lu, "Multiphase CFD Simulation of Solid Propellant Combustion in a Small Gun Chamber," International Journal of Chemical Engineering, vol. 2014, 2014. Article ID 971808. 
[10] E. R. De Kock, G. J. F. Smit and J. H. Knoetze, "Implementation of an Internal Ballistics Module in OpenFOAM ${ }^{\circledR}$," in Proceedings of the 49 ${ }^{\text {th }}$ AIAA/ASME/SAE/ASEE Joint Propulsion Conference, San Jose, US-CA, 2013.

[11] R. Heiser and E. Meineke, "Multidimensional Interior Ballistics Two-Phase Flow and the Chambrage Problem," Journal of Propulsion and Power, vol. 7, no. 6, pp. 909-914, 1991.

[12] E. Meineke and R. Heiser, "A complete numerical solution of the interior ballistics chambrage problem," in Proceedings of the $11^{\text {th }}$ International Symposium on Ballistics, Brussels, Belgium, 1989.

[13] J. Nussbaum, P. Helluy, J.-M. Hérard and B. Baschung, "Multi-Dimensional Two-Phase Flow Modeling Applied to Interior Ballistics," Journal of Applied Mechanics, vol. 78, no. 5, 2011.

[14] J. Nussbaum, P. Helluy, J.-M. Hérard and A. Carrière, "Numerical simulations of gas-particle flows with combustion," Journal of Flow, Turbulence and Combustion, vol. 76, no. 4, pp. 403-417, 2006.

[15] P. S. Gough and F. J. Zwarts, "Modeling Heterogeneous Two-Phase Reacting Flow," AIAA Journal, vol. 17, no. 1, pp. $17-25,1979$.

[16] S. Ergun, "Fluid flow through packed columns," Journal of Chemical Engineering Progress, vol. 48, no. 2, pp. 8994, 1952.

[17] H. Krier, S. Rajan and W. F. van Tessel, "Flame-Spreading and Combustion in Packed Beds of Propellant Grains," AIAA Journal, vol. 14, no. 3, pp. 301-309, 1976.

[18] T. G. Manning, D. Park, K. Klingaman, M. Leadore, B. Homan, E. Liu and J. A. Luoma, "Interior Ballistics of CoLayered Gun Propellant," in Proceedings of the $26^{\text {th }}$ International Symposium on Ballistics, Miami, US-FL, pp. 912921, 2011.

[19] T. Gallouët, J.-M. Hérard and N. Seguin, "Numerical Modeling of Two-Phase Flows Using the Two-Fluid TwoPressure Approach," Mathematical Models and Methods in Applied Science, vol. 14, no. 5, pp. 663-700, 2004.

[20] Q. Qin and X. Zhang, "Numerical investigation on combustion in muzzle flows using an inert gas labelling method," International Journal of Heat and Mass Transfer, vol. 101, no. 1, pp. 91-103, 2016.

[21] H. Childs, E. Brugger, B. Whitlock, J. Meredith, S. Ahern and D. Pugmire, "Visit: An End-User Tool For Visualization and Analyzing Very Large Data," in High Performance Visualization - Enabling Extreme-Scale Scientific Insight, Lawrence Livermore National Laboratory, US-CA, 2012, pp. 357-372.

[22] C. van Driel, M. Straathof and J. van Lingen, "Developments in Additive Manufacturing of Energetic Materials at TNO," in Proceedings of the $30^{\text {th }}$ International Symposium on Ballistics, Long Beach, US-CA, 2017, pp. 862-875. 